\title{
Field theory simulation of Abelian-Higgs cosmic string cusps
}

\author{
Ken D. Olum* and J. J. Blanco-Pillado ${ }^{\dagger}$ \\ Institute of Cosmology \\ Department of Physics and Astronomy \\ Tufts University \\ Medford, MA 02155
}

(December 1998)

\begin{abstract}
We have performed a lattice field theory simulation of cusps in Abelian-Higgs cosmic strings. The results are in accord with the theory that the portion of the strings which overlaps near the cusp is released as radiation. The radius of the string cores which must touch to produce the evaporation is approximately $r=1$ in natural units. In general, the modifications to the string shape due to the cusp may produce many cusps later in the evolution of a string loop, but these later cusps will be much smaller in magnitude and more closely resemble kinks.
\end{abstract}

98.80.Cq 11.27.+d

\section{INTRODUCTION}

Cosmic strings arise naturally in spontaneous symmetry breaking phase transitions in which the vacuum manifold after the transition is not simply connected [1]. (For reviews see [2,3]). A simple field theory which possesses such defects is the Abelian-Higgs model,

$$
\mathcal{L}=D_{\mu} \bar{\phi} D^{\mu} \phi-\frac{1}{4} F_{\mu \nu} F^{\mu \nu}-\frac{\lambda}{4}\left(|\phi|^{2}-\eta^{2}\right)^{2},
$$

with

$$
D_{\mu} \phi=\left(\partial_{\mu}-i e A_{\mu}\right) \phi .
$$

We can choose units such that $\eta=1$ and $e=1$. We will also work in the "critical coupling" regime in which $\beta=\lambda /\left(2 e^{2}\right)=1$ so that in our units $\lambda=2$.

The fields of an infinitely long, static, straight string are given by [4]

$$
\begin{aligned}
\phi(r) & =e^{i \theta} f(r) \\
A_{\theta} & =-\frac{\alpha(r)}{r}
\end{aligned}
$$

where $f(0)=\alpha(0)=0$ and $f(r) \longrightarrow 1$ and $\alpha(r) \longrightarrow 1$ as $r \longrightarrow \infty$. The exact form of $f$ and $\alpha$ must be found numerically.

If the string core radius is small as compared to the radius of curvature of a string, the action can be approximated by the Nambu action,

$$
S=-\mu \int d^{2} \zeta \sqrt{-\operatorname{det} \gamma}
$$

where $\mu$ is the energy per unit string length, $\gamma$ is the metric on the world sheet of the string and $\zeta^{1}, \zeta^{2}$ denote

\footnotetext{
*Email address: kdo@alum.mit.edu

${ }^{\dagger}$ Email address: jose@cosmos2.phy.tufts.edu
}

the world sheet coordinates. We can choose parameters $\sigma$ and $t$ in such a way that the position of the string is given at any time $t$ by a function $\mathbf{x}(\sigma, t)$ that satisfies

$$
\begin{aligned}
\left|\mathbf{x}^{\prime}(\sigma, t)\right|^{2}+|\dot{\mathbf{x}}(\sigma, t)|^{2} & =1 \\
\mathbf{x}^{\prime}(\sigma, t) \cdot \dot{\mathbf{x}}(\sigma, t) & =0 .
\end{aligned}
$$

The equation of motion is

$$
\mathbf{x}^{\prime \prime}(\sigma, t)=\ddot{\mathbf{x}}(\sigma, t),
$$

where $\mathbf{x}^{\prime}$ denotes differentiation with respect to $\sigma$ and $\dot{\mathbf{x}}$ denotes differentiation with respect to $t$. Equation (bb) means that the velocity of the string is always in the plane perpendicular to the string while Eq. (6a) parameterizes the string with constant energy per unit $\sigma$. The general solution of Eqs. (6) and (7) is

$$
\mathbf{x}(\sigma, t)=\frac{1}{2}(\mathbf{a}(\sigma-t)+\mathbf{b}(\sigma+t)),
$$

where $\mathbf{a}$ and $\mathbf{b}$ are arbitrary functions that satisfy $\left|\mathbf{a}^{\prime}\right|=$ $\left|\mathbf{b}^{\prime}\right|=1$. The functions $\mathbf{a}$ and $\mathbf{b}$ describe waves traveling in the positive and negative $\sigma$ directions, respectively.

As long as the string remains smooth, its entire evolution is given by Eq. (8) and the state of the string at all times is fixed by the functions $\mathbf{a}^{\prime}$ and $\mathbf{b}^{\prime}$. The values of these functions are unit vectors, so we can consider them to trace out paths on the surface of the unit sphere. For a string loop, all quantities will be periodic in $\sigma$, so the paths on the unit sphere will be closed. Furthermore, since $\mathbf{a}$ and $\mathbf{b}$ are themselves periodic,

$$
\int d \sigma \mathbf{a}^{\prime}(\sigma)=\int d \sigma \mathbf{b}^{\prime}(\sigma)=0,
$$

which is to say that the center of gravity of the paths of $\mathbf{a}^{\prime}$ and $\mathbf{b}^{\prime}$ is at the center of the sphere. Thus, for example, $\mathbf{a}^{\prime}$ or $\mathbf{b}^{\prime}$ could not lie entirely in a single hemisphere, and, in general, we would expect that there would be one or more crossings between the paths of $\mathbf{a}^{\prime}$ and $\mathbf{b}^{\prime}$. By the same argument, there will be places where $\mathbf{a}^{\prime}$ and 
$-\mathbf{b}^{\prime}$ have the same value, that is to say values of $\sigma_{a}$ and $\sigma_{b}$ at which $\mathbf{a}^{\prime}\left(\sigma_{a}\right)+\mathbf{b}^{\prime}\left(\sigma_{b}\right)=0$. As a result, at time $t=\left(\sigma_{b}-\sigma_{a}\right) / 2$ and position $\sigma=\left(\sigma_{b}+\sigma_{a}\right) / 2$ we will find $\mathbf{x}^{\prime}(\sigma, t)=\left(\mathbf{b}^{\prime}(\sigma+t)+\mathbf{a}^{\prime}(\sigma-t)\right) / 2=0$ and $|\dot{\mathbf{x}}(\sigma, t)|=\mid\left(\mathbf{b}^{\prime}(\sigma+t)-\mathbf{a}^{\prime}(\sigma-t) \mid / 2=1\right.$. Such a point is a cusp. The string there is momentarily moving (in the Nambu-Goto approximation) at the speed of light.

As the string evolves toward the time of the cusp, the Nambu-Goto approximation will break down. Since $\mathbf{x}^{\prime}=$ 0 at the cusp, the segments of string point in the same direction leaving the cusp, and so there is the potential for overlap between the segments on the two sides. When the string cores overlap, the topological constraints which stabilize the string no longer operate, and the energy in the string can be released as radiation. This radiation is of cosmological interest because it could potentially lead to observable cosmic rays, and because it affects the rate at which oscillating loops lose their energy [5 10].

At some point when the cusp-related evolution is complete, the string will once again be smooth and nonoverlapping, and will resume Nambu-Goto evolution with new functions $\mathbf{a}^{\prime}$ and $\mathbf{b}^{\prime}$. One can consider the evolution at the time of the cusp to be a scattering process which takes an initial state of $\mathbf{a}^{\prime}$ and $\mathbf{b}^{\prime}$ and produces a final state with new $\mathbf{a}^{\prime}$ and $\mathbf{b}^{\prime}$ and a certain amount of outgoing radiation.

To determine the amount of radiation emitted, and the resulting configuration of the string, we have written a lattice field theory simulation of the Abelian-Higgs field theory.

\section{THEORETICAL EXPECTATIONS}

The usual expectation is that the string can participate in cusp evaporation when the "cores" of the strings on the two sides of the cusp are overlapping. A parameter $r$ gives the "core radius," i.e., the point at which overlap dynamics can begin, as shown in Fig. 11. We expect this radius to be on the order of $\eta^{-1}=1$ in our units.

In 10 we showed that a generic cusp can be analyzed in a frame in which $\mathbf{x}_{0}^{\prime \prime \prime}$ is parallel to $\dot{\mathbf{x}}_{0}$, where the subscript 0 denotes quantities evaluated at the cusp. Throughout this paper we will work in such a reference frame.

In section III of [10] we calculated the amount of overlap between the two branches of a string at a cusp. The result was that the two segments of the string overlap from the cusp out to a distance $\sigma_{c}$ given by

$$
\sigma_{c}=\left(3 r \sqrt{\frac{\left|\mathbf{x}_{0}^{\prime \prime}\right|^{2}+\left|\dot{\mathbf{x}}_{0}^{\prime}\right|^{2}}{\left|\dot{\mathbf{x}}_{0}^{\prime} \cdot \mathbf{x}_{0}^{\prime \prime}\right|^{2}}-\frac{1}{\left|\mathbf{x}_{0}^{\prime \prime}\right|^{2}}}\right)^{1 / 2}+O(r)
$$

We expect an amount of string of total length $2 \sigma_{c}$ to be replaced by a short bridging string of length $2 r$ or less, so that the energy released in radiation will be approximately $2 \mu \sigma_{c}$.

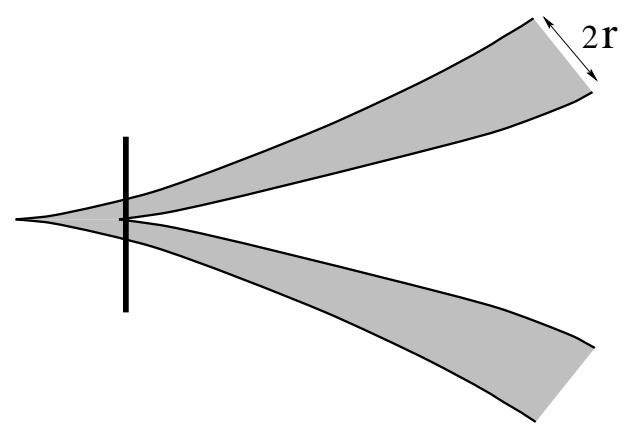

FIG. 1. Schematic shape for a cusp in a cosmic string. The shaded region corresponds to the core of the string. To the left of the vertical line, the cores overlap, and the energy could be released as radiation. The string appears thinner near the cusp because of Lorentz contraction.

In a string of cosmological size, $\sigma_{c}$ would be very much larger than $r$, and the approximation in Eq. (10) would be very accurate. However, we cannot simulate a string with such a large ratio of length scales. (Our largest simulation has $\sigma_{c} / r \approx 17$.) To improve the accuracy of our prediction, we proceed as follows. We assume that the string has Nambu-Goto evolution until the time of the cusp (which is confirmed by simulation, see below), and compute the positions $\sigma_{+}$and $\sigma_{-}$on the two sides of the cusp at which the (Lorentz contracted) string cores just touch. It is possible that $\sigma_{+}$and $\sigma_{-}$are not uniquely defined by this condition, since perhaps $\sigma_{+}$could be moved away from the cusp while $\sigma_{-}$was moved toward the cusp to produce different points of overlap. In this case, we take the points which lead to the largest emission of energy. We then assume that the string segment from $\sigma_{-}$to $\sigma_{+}$will be replaced by a bridging string at rest between $\mathbf{x}\left(\sigma_{-}\right)$and $\mathbf{x}\left(\sigma_{+}\right)$, to arrive at an estimate of the energy release. Since we do not know the radius $r$ at which overlap dynamics become possible, we can consider the simulation to provide us with a value for this radius.

How should the paths of $\mathbf{a}^{\prime}$ and $\mathbf{b}^{\prime}$ be affected by the evaporation of the cusp? Near the cusp, $\mathbf{a}^{\prime}(\sigma) \sim \mathbf{a}_{0}^{\prime}=$ $-\dot{\mathbf{x}}_{0}$ and $\mathbf{b}^{\prime}(\sigma) \sim \mathbf{b}_{0}^{\prime}=\dot{\mathbf{x}}_{0}$. Outside the region of overlap, they will retain these values, but inside that region we will have a bridging string. The bridging string will point in the direction of $\mathbf{x}_{0}^{\prime \prime \prime}$ because that is the direction in which the two arms of the cusp spread apart. Since the motion of the string near the cusp is approximately $\dot{\mathbf{x}}_{0}$, which is parallel to $\mathbf{x}_{0}^{\prime \prime \prime}$, the bridging string will be created approximately stationary. Thus on the bridge, $\mathbf{a}^{\prime}(\sigma)=$ $\mathbf{b}^{\prime}(\sigma) \propto \mathbf{x}_{0}^{\prime \prime \prime}$ with a positive constant of proportionality. There are thus two cases, depending on whether $\mathbf{x}_{0}^{\prime \prime \prime}$ is parallel or antiparallel to $\dot{\mathbf{x}}_{0}$. If they are parallel, then $\mathbf{b}^{\prime}(\sigma)$ on the bridge will have roughly the same value that it has on nearby unaffected portions of the string, but $\mathbf{a}^{\prime}(\sigma)$ on the bridge will have nearly the opposite value as 


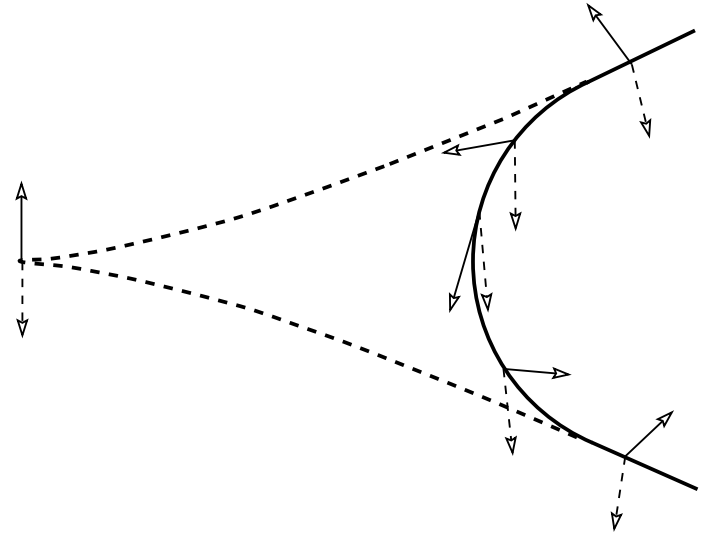

FIG. 2. Schematic of the evolution of the functions $\mathbf{a}^{\prime}$ (solid arrows) and $\mathbf{b}^{\prime}$ (dashed arrows) before (dashed line) and after (solid line) the cusp evaporation process.

it does nearby, as shown in Fig. 2. Thus in this case we expect the path of $\mathbf{a}^{\prime}(\sigma)$ to be broken near the point of crossing and reconnected around the opposite side of the unit sphere. In the case that $\mathbf{x}_{0}^{\prime \prime \prime}$ and $\dot{\mathbf{x}}_{0}$ are antiparallel, we will have the reverse behavior. The path of $\mathbf{a}^{\prime}(\sigma)$ will be pretty much unaffected, while $\mathbf{b}^{\prime}(\sigma)$ will be broken and reconnected. This effect of the cusp on the evolution of the functions $\mathbf{a}^{\prime}$ and $\mathbf{b}^{\prime}$ on the unit sphere is shown in Figs. 3 and 4 .

\section{SIMULATION}

\section{A. Lattice Action}

To put Eq. (11) on a lattice, we have used a somewhat different approach from those that have been used in the past 11 13]. We put the system on a lattice in both space and time, with the scalar field $\phi$ stored on the vertices of the lattice, the vector field $A_{\mu}$ on the links, and the field strength $F_{\mu \nu}$ on the faces. The action can be specified as follows.

Let $s$ denote a lattice site, $l$ a directed link from site $l_{-}$to site $l_{+}$, and $f$ a directed face made of links $f_{1}, f_{2}$, $f_{3}$, and $f_{4}$. Let $\sum_{s}$ denote summation over all sites, $\sum_{l}$ summation over all links, counting each link once, and $\sum_{f}$ summation over all faces, counting each face once. Let $\sum_{l \mid l_{+}=s}$ denote summation over all links that end in site $s$ and $\sum_{f \mid f_{1}=l}$ denote summation over all faces that border link $l$. Then the lattice action is

$$
S=\Delta x^{3} \Delta t\left(\sum_{s} \mathcal{L}_{s}+\sum_{l} \mathcal{L}_{l}+\sum_{f} \mathcal{L}_{f}\right)
$$

where

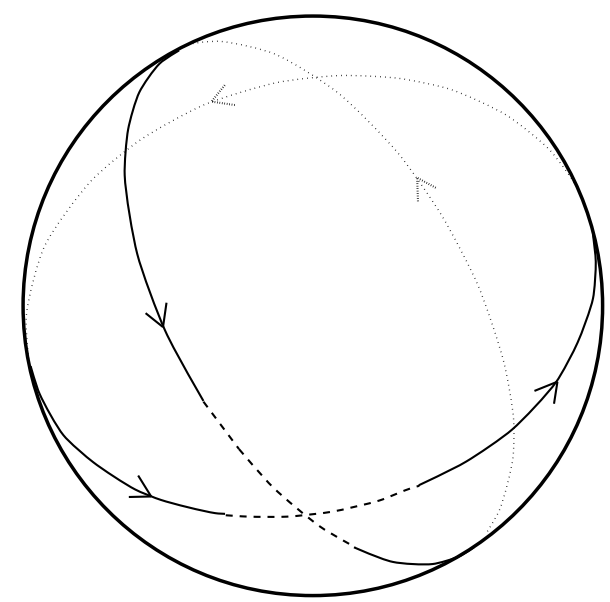

FIG. 3. The paths of $\mathbf{a}^{\prime}$ and $-\mathbf{b}^{\prime}$ before the cusp. The dashed segments are the parts that will be affected by the cusp. The dotted curves are the paths around the back of the sphere.

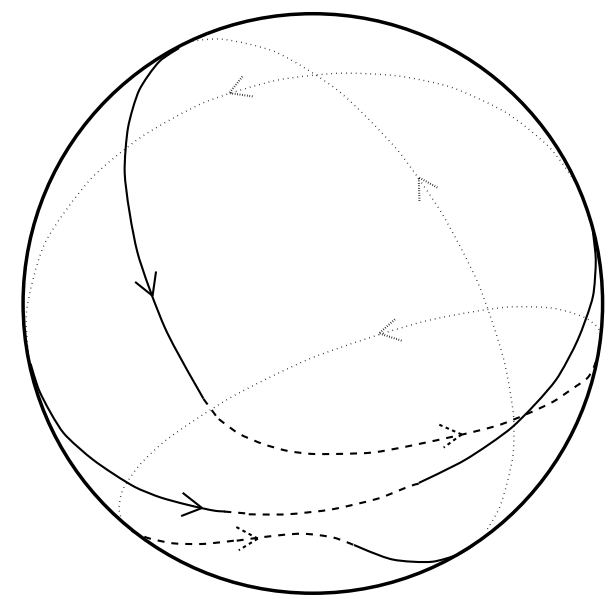

FIG. 4. The paths of $\mathbf{a}^{\prime}$ and $-\mathbf{b}^{\prime}$ after the cusp. One of the paths has been broken and reconnected around the back of the sphere. 


$$
\begin{aligned}
\mathcal{L}_{s} & =-V\left(\phi_{s}\right)=-\frac{1}{4} \lambda\left(\left|\phi_{s}\right|^{2}-\eta^{2}\right)^{2} \\
\mathcal{L}_{l} & =g_{l} D_{l} \bar{\phi} D_{l} \phi \\
\mathcal{L}_{f} & =-\frac{1}{2} g_{f} F_{f}^{2} .
\end{aligned}
$$

Here $\Delta x$ and $\Delta t$ are the spatial and temporal lattice spacing; $g_{l}$ is the metric, $g_{l}=+1$ if $l$ is timelike, and $g_{l}=-1$ if $l$ is spacelike; and $g_{f}=g_{f_{1}} g_{f_{2}}$, i.e., $g_{f}=+1$ if all sides of $f$ are spacelike, and $g_{f}=-1$ if $f$ has a timelike side. The discrete covariant derivative is given by

$$
\begin{aligned}
D_{l} \phi & =\Delta_{l}\left(\phi_{l_{+}}-e^{i \Delta_{l} e A_{l}} \phi_{l_{-}}\right) \\
D_{l} \bar{\phi} & =\Delta_{l}\left(\bar{\phi}_{l_{+}}-e^{-i \Delta_{l} e A_{l}} \bar{\phi}_{l_{-}}\right)
\end{aligned}
$$

where $\Delta_{l}$ denotes $\Delta x$ if $l$ is spacelike or $\Delta t$ if $l$ is timelike. The field strength is given by

$$
F_{f}=\Delta_{f_{1}}^{-1} \Delta_{f_{2}}^{-1}\left(\Delta_{f_{1}} A_{f_{1}}+\Delta_{f_{2}} A_{f_{2}}+\Delta_{f_{3}} A_{f_{3}}+\Delta_{f_{4}} A_{f_{4}}\right) .
$$

The action of Eq. (11) is invariant under a discrete gauge transformation given by a number $\Lambda_{s}$ at each lattice site,

$$
\begin{aligned}
& \phi_{s} \longrightarrow e^{i \Lambda_{s}} \phi_{s} \\
& A_{l} \longrightarrow A_{l}+\Delta_{l}^{-1}\left(\Lambda_{l_{+}}-\Lambda_{l_{-}}\right) .
\end{aligned}
$$

We will work in temporal gauge, $A_{l}=0$ for all timelike links $l$, which greatly simplifies the calculation.

The equations of motion resulting from Eq. (11) are

$$
\begin{aligned}
\sum_{l \mid l_{+}=s} g_{l} \Delta_{l}^{-2}\left(\bar{\phi}_{l_{-}} e^{-i \Delta_{l} e A_{l}}-\bar{\phi}_{s}\right)+\frac{\partial V}{\partial \phi_{s}} & =0 \\
\sum_{f \mid f_{1}=l} \Delta_{f_{2}}^{-1} g_{f} F_{f} & =j_{l}
\end{aligned}
$$

where the link current is

$$
j_{l}=i e \Delta_{l}^{-1} g_{l}\left(e^{-i e \Delta_{l} A_{l}} \bar{\phi}_{l_{-}} \phi_{l_{+}}-e^{i e \Delta_{l} A_{l}} \bar{\phi}_{l_{+}} \phi_{l_{-}}\right) .
$$

The stability of our finite difference scheme requires the Courant condition, $\Delta t / \Delta x<1 / \sqrt{3} \approx 0.58$ [14], for three spatial dimensions. In fact, ratios slightly less than this are still unstable in our code, presumably due to numerical error. To be safe, we have used $\Delta t / \Delta x=0.5$. To verify that this condition is sufficient, we have checked that our simulations conserve energy over long periods of time.

We have used reflecting boundary conditions (fixed field values) on the top and the bottom faces of our lattice box, and first order absorbing boundary conditions 115,16] on the lateral faces. The absorbing conditions prevent the radiation emitted at the cusp from reflecting off the walls and interacting with the later evolution of the string.

\section{B. Initial Conditions}

To simulate cusp formation we must have an initial field configuration which will evolve into a cusp. This can be done exactly, using a result of Vachaspati et al. 17] that a traveling wave along a straight string is an exact solution of the field theory equations of motion. In other words, an arbitrarily shaped wiggle that propagates at the speed of light along a straight string will not radiate and will retain its form.

These wiggles can be seen also in terms of functions $\mathbf{a}^{\prime}$ and $\mathbf{b}^{\prime}$ in the Nambu-Goto approximation. For example, a straight part of the string in the $\hat{\mathbf{z}}$ direction can be written in terms of $\mathbf{a}^{\prime}=\hat{\mathbf{z}}$ and $\mathbf{b}^{\prime}=\hat{\mathbf{z}}$. Introducing a wiggle traveling in one direction along the string will modify one of the functions $\mathbf{a}^{\prime}$ or $\mathbf{b}^{\prime}$ depending on the direction of the movement of the wiggle, keeping the other function fixed.

Using this method we can obtain the Nambu-Goto description for two wiggles traveling toward each other along a straight string. Then by using [17] we can write down the expression for the fields in terms of the functions $\mathbf{a}^{\prime}$ and $\mathbf{b}^{\prime}$ and the static string fields given by Eqs. (3) and (4). By calculating the values for the fields when the two wiggles are still separated by a portion of straight string we ensure that our initial conditions are exact, so we do not have to use a relaxation procedure as is necessary in other field theory simulation schemes 11 13.

We design the two colliding wiggles such that they will produce a cusp in the Nambu-Goto evolution and use them as our initial conditions in the field theory code. One could imagine that the string would evolve into a different configuration due to interaction before the time of the cusp, but as we will show later, that does not occur: the string core in the field theory simulation follows the path that the Nambu-Goto evolution predicts until immediately before the cusp.

As described earlier, the cusp is characterized in the Nambu-Goto approximation by the intersection of $\mathbf{a}^{\prime}$ and $-\mathbf{b}^{\prime}$ on the unit sphere. Far outside the area of interaction, the string will be straight with $\mathbf{a}^{\prime}$ at the north pole of the unit sphere, and $-\mathbf{b}^{\prime}$ at the south pole. Thus we are looking for functions $\mathbf{a}^{\prime}$ and $\mathbf{b}^{\prime}$ which make loops on the sphere, starting and finishing at the poles and crossing at some point of the sphere.

Two closed loops cannot intersect in just a single point, unless it is a point of tangency, which does not give rise to a generic cusp. But we want to obtain information about the energy radiated by just one cusp, so we do not want to have any interference in the dynamics due to the formation of a second one. (A realistic cosmological string would have multiple cusps, but they would be very well separated.) To prevent this interference we will design the two wiggles so that the first cusp will alter the string in such a way that the second one will not occur. We will choose our parameters so that $\mathbf{x}_{0}^{\prime \prime \prime} \cdot \dot{\mathbf{x}}_{0}>0$, so we expect $\mathbf{a}^{\prime}$ to be broken and reconnected, while the path 
of $\mathbf{b}^{\prime}$ will not be greatly affected. Thus we will arrange for $\mathbf{b}^{\prime}$ to cross $\mathbf{a}^{\prime}$ twice at the same point, so that when $\mathbf{a}^{\prime}$ is reconnected to eliminate the first crossing, the second crossing will be eliminated as well. We will choose a simple path for $\mathbf{b}^{\prime}$ that starts at the pole, runs along a meridian, crosses $\mathbf{a}^{\prime}$ at the equator, and continues for short distance before turning around and returning along the same path.

We must also arrange that the magnitudes of $\mathbf{a}_{0}^{\prime \prime}$ and $\mathbf{b}_{0}^{\prime \prime}$ are different to avoid a pathological situation which leads to extra energy emission, as discussed in [10]. We also want to work in the canonical frame where $\mathbf{x}_{0}^{\prime \prime \prime}$ is parallel to $\dot{\mathbf{x}}_{0}$, so we require that $\mathbf{a}_{0}^{\prime \prime \prime}+\mathbf{b}_{0}^{\prime \prime \prime}$ is perpendicular to $\mathbf{a}_{0}^{\prime \prime}$ and to $\mathbf{b}_{0}^{\prime \prime}$.

We will start by specifying our desired values for $\mathbf{a}_{0}^{\prime \prime}$ and $\mathbf{a}_{0}^{\prime \prime \prime}$. As much as possible we would like other derivatives not to contribute near the cusp, so we will set

$$
\mathbf{a}_{\text {unnormalized }}^{\prime}(\sigma)=\mathbf{a}_{0}^{\prime}+\sigma \mathbf{a}_{0}^{\prime \prime}+\frac{\sigma^{2}}{2} \mathbf{a}_{0}^{\prime \prime \prime}
$$

and then

$$
\mathbf{a}^{\prime}(\sigma)=\frac{\mathbf{a}_{\text {unnormalized }}^{\prime}(\sigma)}{\left|\mathbf{a}_{\text {unnormalized }}^{\prime}(\sigma)\right|}
$$

We will use this form for $\sigma \in\left[-\sigma_{1}, \sigma_{1}\right]$, where $\sigma_{1}$ is chosen to be large enough that changes in $\mathbf{a}^{\prime}$ outside this range will not have too much effect on the cusp dynamics.

Outside of this range, we just have to connect $\mathbf{a}^{\prime}$ to the north pole of the unit sphere. We do this by choosing paths of circles on the sphere which will smoothly interpolate between $\mathbf{a}^{\prime}\left(-\sigma_{1}\right)$ and $\mathbf{a}^{\prime}\left(\sigma_{1}\right)$ and the pole.

The result of this design for $\mathbf{a}^{\prime}$ and $-\mathbf{b}^{\prime}$ is shown in Fig. 5 .

To produce the initial field configuration, we offset the wiggles produced by $\mathbf{a}^{\prime}$ and $\mathbf{b}^{\prime}$, so that one is at the top of the lattice moving downward and other is at the bottom of the lattice moving upward. The actual shape of the string can be seen in Fig. 6

When the two wiggles combine, we will look at the shape of the string at the time of the cusp in the NambuGoto approximation, and define the "length scale" of the cusp for the sake of discussion as

$$
L=\frac{1}{\left|\mathbf{x}_{0}^{\prime \prime}\right|} \text {. }
$$

\section{Recovering the energy and form of the string.}

In order to obtain the amount of energy released in the cusp evaporation, we implemented a method to recover, out of the field theory simulation, the total amount of energy left in the string.

We first identify the plaquettes of the lattice that the string goes through, by looking for places where the phase of the complex scalar field on the four corners of the

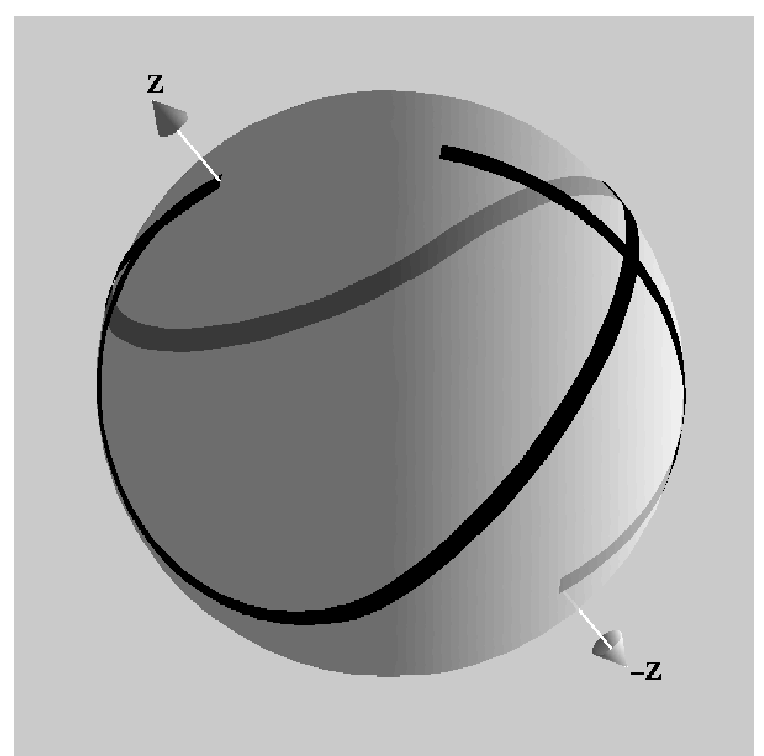

FIG. 5. Initial conditions for the functions $\mathbf{a}^{\prime}$ and $-\mathbf{b}^{\prime}$, on the unit sphere.

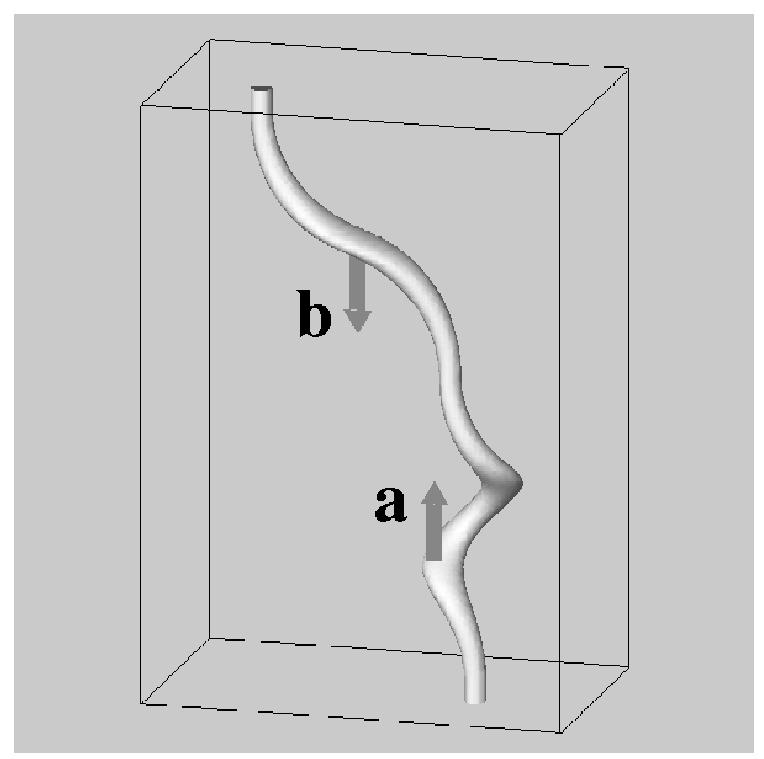

FIG. 6. The initial conditions. The surface shown is a surface of constant energy density surrounding the string core. 
plaquette wraps around the origin in the complex plane. As long as two strings do not go through the same lattice cube, this technique always allows the string to be traced from one face to the next. We take the path of the string to go through the centers of these plaquettes. (We have tried some algorithms for improving the path of the string by closer examination of the field values at the corners of the plaquettes, but they did not produce significantly better results.)

This technique produces a discrete approximation to the string path, which we then smooth by a Gaussian convolution. The end result is a list of smoothed strings at different times, parameterized by an arbitrary parameter $\lambda$. We will denote these string positions by $\mathbf{x}(\lambda, t)$.

Near the time and position of the cusp, the dynamics are not well described by the motion of a string, but rather involve the complete field configuration. One can still recover a path of faces around which the field phase winds, but the different positions of this path at different times do not represent motion, but rather the unraveling of a field configuration. However, since we are only interested in the difference between the energy in the string before and after the cusp, we will just apply this method at times well before or well after the cusp where we are certain that the Nambu approximation is a faithful description of the string.

To recover the energy of the string we first calculate $\dot{\mathbf{x}}(\lambda, t)$ by computing

$$
\dot{\mathbf{x}}(\lambda, t)=\frac{\mathbf{x}(\lambda, t)-\mathbf{x}\left(\lambda^{\prime}, t-N \Delta t\right)}{N \Delta t}
$$

where $N$ is a number of lattice time steps over which we average to reduce the effect of errors in recovering the string position, and $\lambda^{\prime}$ is determined by the condition that $\left(\mathbf{x}(\lambda, t)-\mathbf{x}\left(\lambda^{\prime}, t-N \Delta t\right)\right)$ be perpendicular to $d \mathbf{x}(\lambda, t) / d \lambda$.

Once we have calculated the velocity we can obtain the relation between $\lambda$ and the usual parameter $\sigma$ by using Eq. (6a),

$$
\left|\frac{d \mathbf{x}(\lambda, t)}{d \lambda}\right| \frac{d \lambda}{d \sigma}=\sqrt{1-|\dot{\mathbf{x}}(\lambda, t)|^{2}}
$$

So

$$
d \sigma=\frac{d \lambda}{\sqrt{1-|\dot{\mathbf{x}}(\lambda, t)|^{2}}}\left|\frac{d \mathbf{x}(\lambda, t)}{d \lambda}\right| .
$$

The total length of string is just the integral of the equation above and so the energy is

$$
E_{\text {string }}=\mu \int d \sigma \text {. }
$$

Once we have obtained the vectors $\mathbf{x}^{\prime}$ and $\dot{\mathbf{x}}$ for the string, we can get the functions, $\mathbf{a}^{\prime}(\sigma, t)$, and $\mathbf{b}^{\prime}(\sigma, t)$ from the relations

$$
\begin{aligned}
\mathbf{a}^{\prime}(\sigma, t) & =\mathbf{x}^{\prime}(\sigma, t)-\dot{\mathbf{x}}(\sigma, t) \\
\mathbf{b}^{\prime}(\sigma, t) & =\mathbf{x}^{\prime}(\sigma, t)+\dot{\mathbf{x}}(\sigma, t)
\end{aligned}
$$

and study the change of these functions due to the cusp dynamics.

\section{RESULTS}

We ran the simulation code for cusp scales (as defined by Eq. (22)) from $L \approx 5$ up to $L \approx 34$. For sizes up to $L \approx 17$ we used $\Delta x=0.25$ or less, which gives a quite accurate simulation. For larger sizes we were forced by memory constraints to use larger values of $\Delta x$ up to $\Delta x=0.425$. These larger values produce somewhat decreased accuracy, especially in those parts of the string which are rapidly moving. They also reduce the accuracy with which the position of the string and thus the energy emission can be recovered from the simulation. However, we feel that these simulations are still sufficiently accurate to draw some general conclusions about the cusp evaporation process.

The code was written in Lisp and executed on a Digital Alpha processor. The largest lattices required about 700 $\mathrm{MB}$ of memory and one week of CPU time.

We first run the code letting only one wiggle evolve on the straight string, setting $\mathbf{b}^{\prime}=\hat{\mathbf{z}}$. This experiment confirms Vachaspati's result [17], showing the traveling wave on the string without any radiation. This is also a check for the code, since it shows stability and energy conservation for the evolution with the boundary conditions specified above.

With the two colliding wiggles, the string forms a cusp as expected. Energy is conserved to within 1\% until the radiation emitted at the cusp begins to be absorbed at the boundary. Figures 7 and 8 show the string shortly after the moment at which the Nambu-Goto approximation predicts the cusp. Except near the cusp, the NambuGoto evolution has been followed accurately. But near the cusp, the core of the string has collapsed away from the area where the cusp energy is stored and has released this energy in the form of radiation. This is what one would expect from the overlap model. At some point near the cusp the strings are close enough that the fields can untangle, and the cusp beyond this point is replaced by a bridging string. After this time, the released radiation travels freely in an expanding shell as shown in Fig. 9.

For each simulation run, we recover the shape of the string, the form of $\mathbf{a}^{\prime}$ and $\mathbf{b}^{\prime}$, and the total length of string at each time step. Figure 10 shows a typical graph of the recovered points. There is significant variation in the individual points, both random variation due to the way in which the string core cuts through the lattice, and systematic variation due to inaccuracies in recovering the energy that depend on the string shape. However, there is a clear difference between the string length before and after the time of the cusp. (At times very near the cusp the energy cannot be recovered so there are no corresponding points on the plot.) 


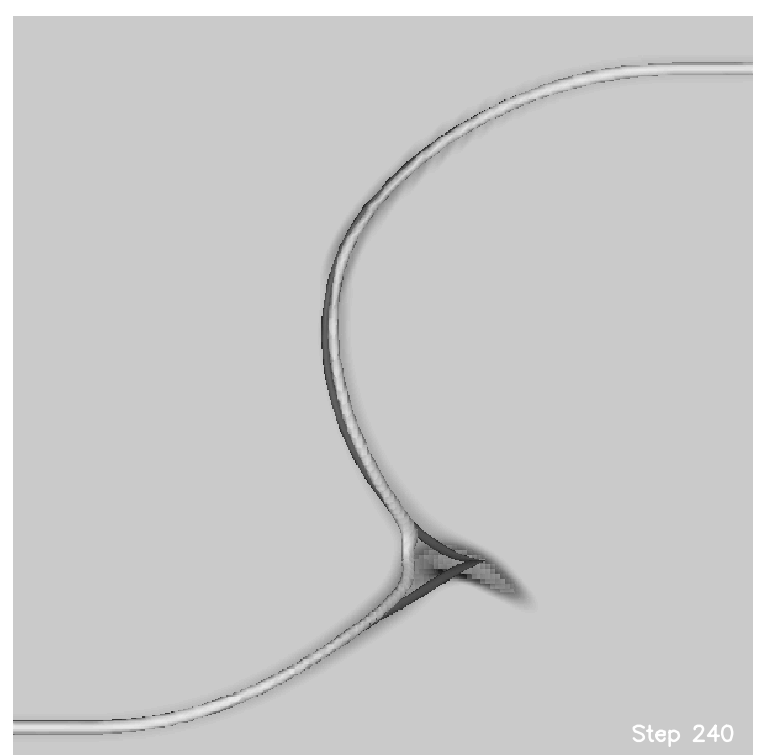

FIG. 7. String evolution at the cusp for $L \approx 17$. The core of the string in the field theory simulation is shown light, and the Nambu-Goto prediction dark. The "fog" shows areas of high energy density. The string core has collapsed away from the point of the cusp, leaving the energy behind in radiation.

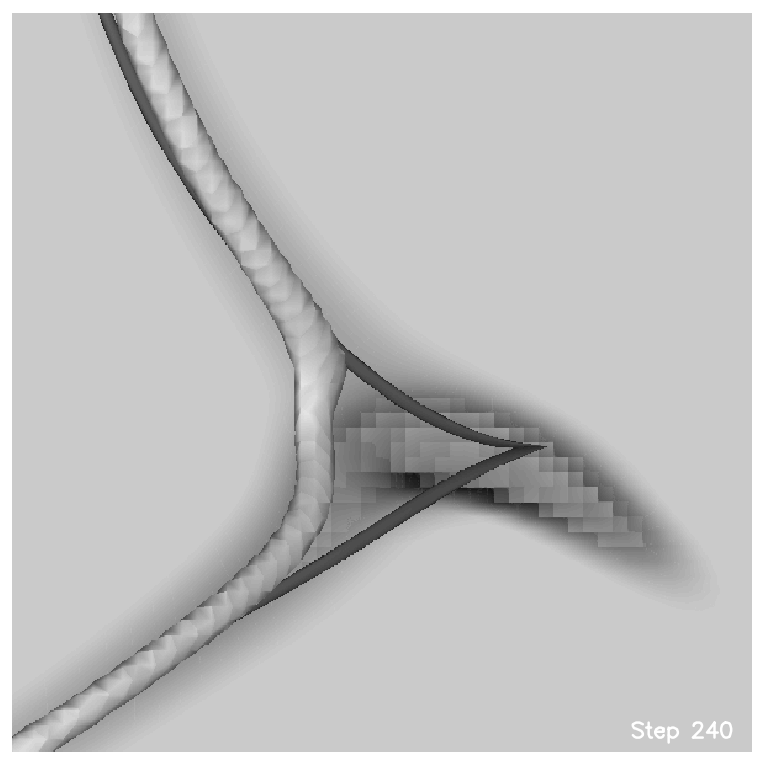

FIG. 8. Close-up view of Fig. 7

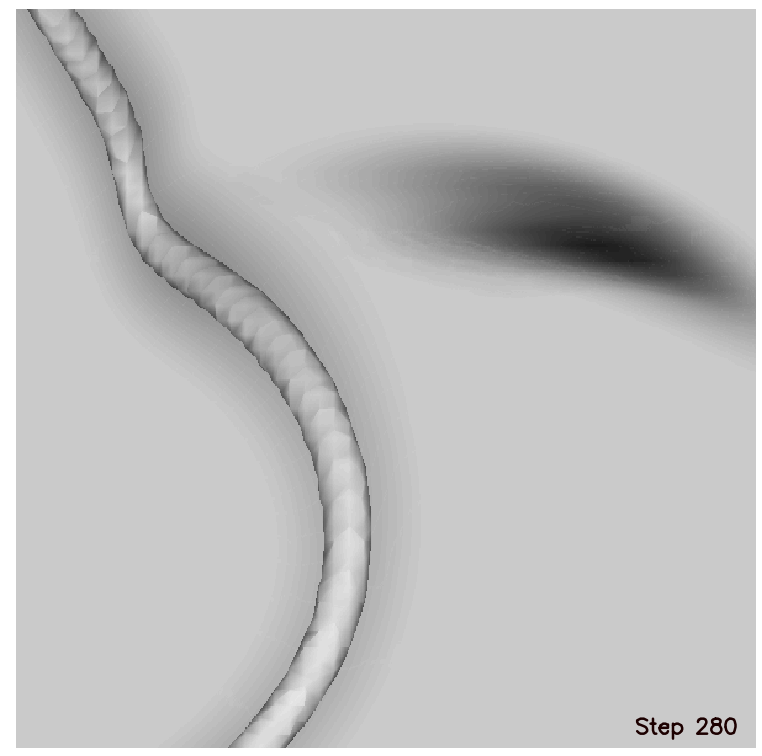

FIG. 9. Close-up view of the core of the string and the radiation after the cusp evaporation. The cloud of radiated energy travels in an expanding shell.

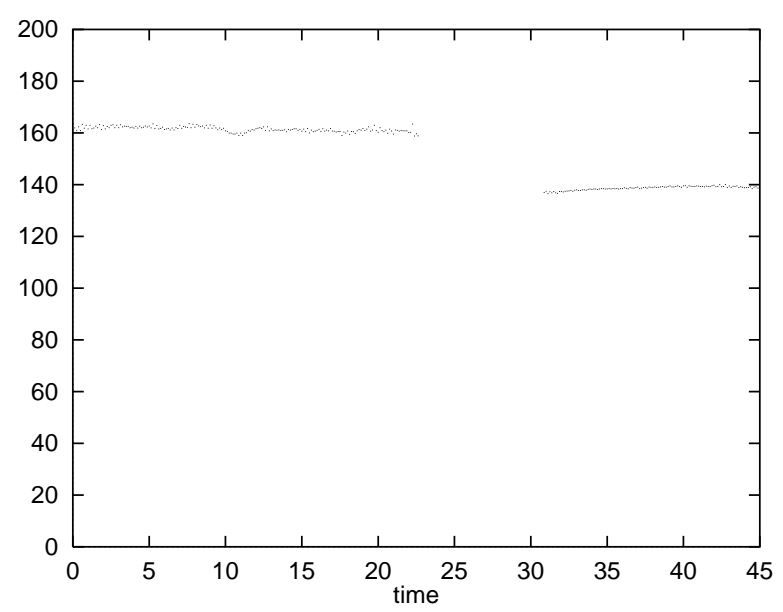

FIG. 10. Recovery of the total amount of $\sigma$ on the string during a cusp evaporation, for $L \approx 17$. Points at left are before the cusp; points at right are those after cusp evaporation. Near the time of the cusp, $\sigma$ cannot be recovered. 


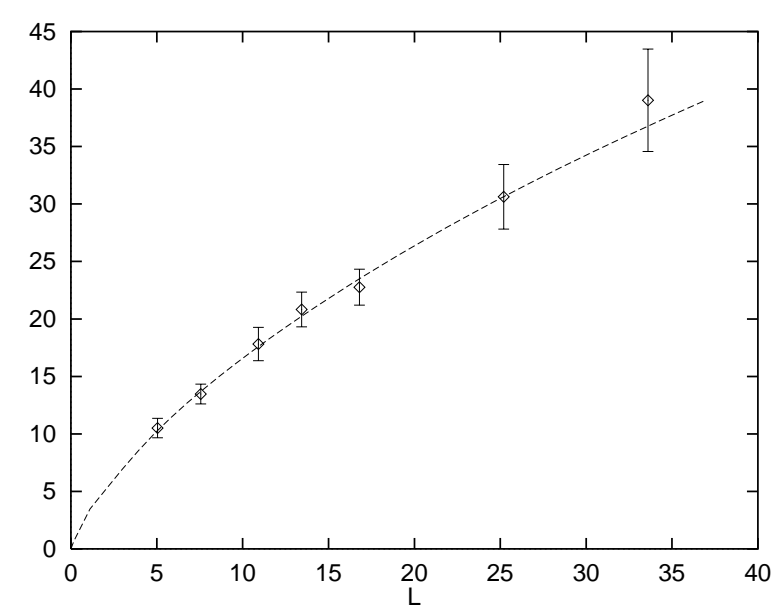

FIG. 11. Amount of $\sigma$ as a function of the length scale $L$. The dashed line corresponds to the theoretical prediction assuming $r=1.15$.

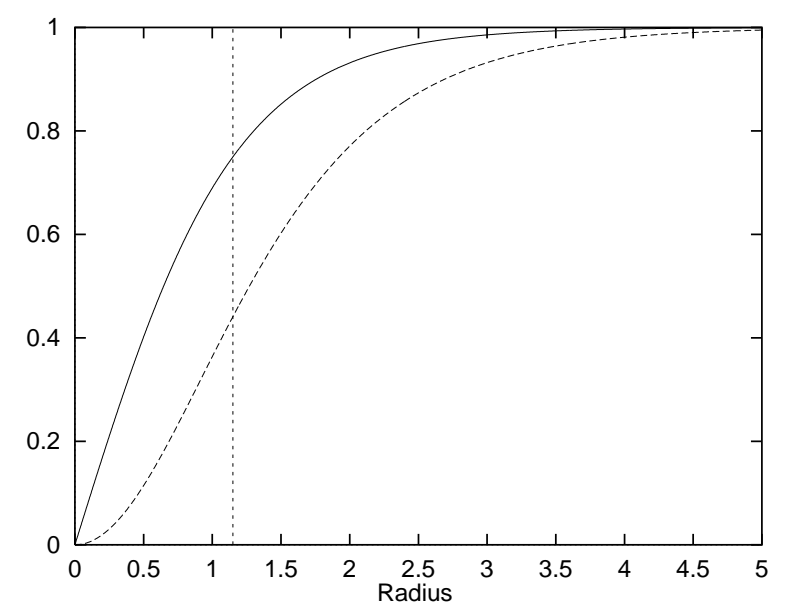

FIG. 12. Functions $f$ (solid curve) and $\alpha$ (dashed curve) for the static straight string. The vertical line indicates the best-fit value of $r$ from the field theory simulation.

In Fig. 11 we plot the string length released by the cusp and compare with theoretical predictions. The error bars in Fig. 11 are a combination of the variation in the actual recovered energy with an estimate of the systematic errors due to the recovery process. As shown, the results are in accord with the overlap model with overlap radius $r \approx 1.15$. Figure 12 shows this value of $r$ as compared to the shape of the string profile. One would expect the overlap dynamics to begin at a radius where the fields have values intermediate between the core values and those at large distances, and the result seems quite reasonable in this regard.

The recovered $\mathbf{a}^{\prime}$ and $-\mathbf{b}^{\prime}$ shortly after the cusp are shown in Fig. 13. By comparison with Fig. 月, we can see that the path of $-\mathbf{b}^{\prime}$ is roughly the same before and after the cusp, but the path of $\mathbf{a}^{\prime}$ has been greatly altered. Far

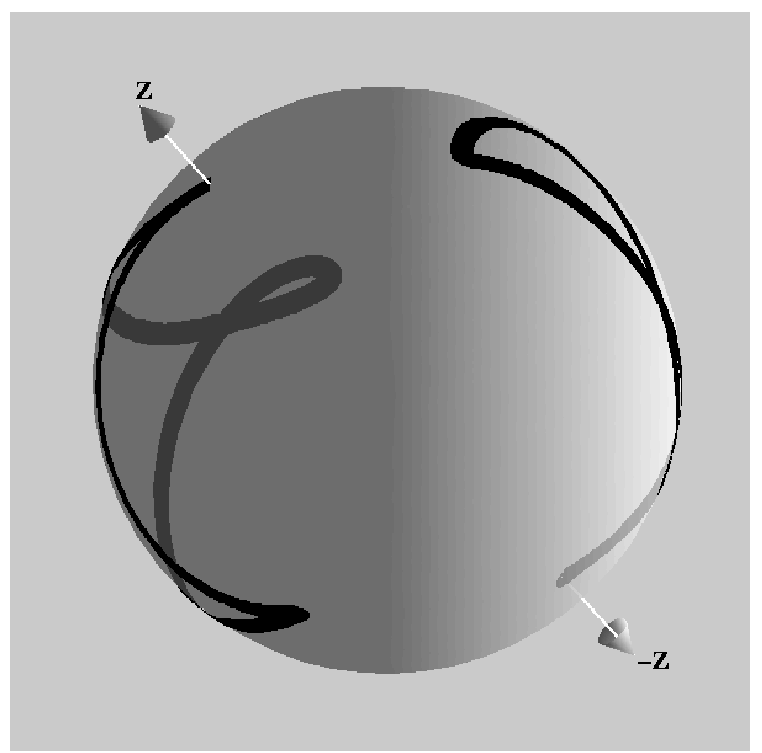

FIG. 13. Recovered functions $\mathbf{a}^{\prime}$ and $-\mathbf{b}^{\prime}$ after the cusp, for $L \approx 17$.

from the cusp it is the same, but in the region of interaction, it has been reconnected around the other side of the sphere from the original crossing point. This reconnection, as planned, prevents the occurrence of a second cusp.

\section{DISCUSSION}

We have simulated a cosmic string cusp in AbelianHiggs field theory on the lattice. Our results are in accord with the model that the evolution is accurately given by the Nambu-Goto equations of motion until nearly the time of the cusp. The effect of the cusp is to release an amount of energy which is well approximated by the overlap model with $r \approx 1.15$.

As discussed in [10], for a string of cosmological size, this model gives an energy emission of order $\mu \sqrt{r L}$, where $L$ is the typical length scale. This is smaller than previous estimates [5 9 by a factor of $(r / L)^{1 / 6}$ and would not lead to observable cosmic rays.

The effect of the evolution through the cusp is to disconnect the path of either $\mathbf{a}^{\prime}$ and $-\mathbf{b}^{\prime}$ and reconnect it around the other side of the unit sphere. We will suppose without loss of generality that $\mathbf{x}_{0}^{\prime \prime \prime} \cdot \dot{\mathbf{x}}_{0}>0$ so that it is $\mathbf{a}^{\prime}$ that is reconnected, while $-\mathbf{b}^{\prime}$ merely has a section deleted and forms a small kink. It is likely that the reconnected $\mathbf{a}^{\prime}$ will have many crossings with $-\mathbf{b}^{\prime}$ and thus might lead to many cusps in the future. However, the total amount of string length in which $\mathbf{a}^{\prime}$ loops around the sphere is quite small, on the order of $r$. Thus the remaining feature involves a bending of the string in about a string radius, and is thus more like a kink than a region of string which would produce a cusp. Any cusps 
in which it is involved will have even smaller emission of radiation than the original cusp.

This result could perhaps be modified by gravitational radiation, which smoothes out small-scale features [18]. Gravitational back-reaction might smooth out the region where $\mathbf{a}^{\prime}$ varies rapidly and restore it to its original crossing with $-\mathbf{b}^{\prime}$. This would produce another cusp at the same position in a subsequent oscillation. However, the region of overlap contains much less energy than before, because most of it has been emitted, so that $\mathbf{a}^{\prime}$ and $-\mathbf{b}^{\prime}$ cross this region in a small amount of $\sigma$. Subsequent gravitational effects might increase this energy, but it will not grow as large as in the original cusp, and so will not lead to observable radiation, as discussed above.

\section{ACKNOWLEDGEMENTS}

We would like to thank Inyong Cho for providing us with a static string profile, and Xavier Siemens and Alex Vilenkin for helpful conversations. This work was supported in part by funding provided by the National Science Foundation. J. J. B. P. is supported in part by the Fundación Pedro Barrie de la Maza.

[1] T. W. B. Kibble, J. Phys. A9, 1387 (1976).

[2] A. Vilenkin and E. P. S. Shellard, Cosmic Strings and other Topological Defects. (Cambridge University Press, Cambridge, 1994).

[3] M. B. Hindmarsh and T. W. B. Kibble, Rep. Prog. Phys. 58, 477 (1995).

[4] H. B. Nielsen and P. Olesen, Nucl. Phys. B61, 45 (1973).

[5] R. H. Brandenberger, Nucl. Phys. B293, 812 (1987).

[6] P. Bhattacharjee, Phys. Rev. D 40, 3968 (1989).

[7] J. H. MacGibbon and R. H. Brandenberger, Nucl. Phys. B331, 153 (1990).

[8] J. H. MacGibbon and R. H. Brandenberger, Phys. Rev. D 47, 2283 (1993).

[9] R. H. Brandenberger, A. T. Sornborger, and M. Trodden, Phys. Rev. D 48, 940 (1993).

[10] J. J. Blanco-Pillado and K. D. Olum, gr-qc/9810005 (unpublished).

[11] K. J. M. Moriarty, E. Myers, and C. Rebbi, Phys. Lett. B 207, 411 (1988).

[12] G. Vincent, N. D. Antunes, and M. Hindmarsh, Phys. Rev. Lett. 80, 2277 (1998).

[13] J. N. Moore and E. P. S. Shellard, astro-ph/9808336 (unpublished).

[14] W. H. Press, S. A. Teukolsky, W. T. Vetterling, and B. P. Flannery, Numerical Recipes in C. The Art of Scientific Computing. (Cambridge University Press, Cambridge, 1992), Vol. 1.

[15] B. Engquist and A. Majda, Math. Comput. 31, 629 (1977).
[16] B. Engquist and L. Halpren, Math. Meth. Appl. Sci. 13, 189 (1990).

[17] Vachaspati and T. Vachaspati, Phys. Lett. B 41, 238 (1990).

[18] J. M. Quashnock and D. N. Spergel, Phys. Rev. D 42, 2505 (1990). 